\title{
Aplicação da Medida de Independência Funcional na prática do autocuidado em uma unidade de internação geriátrica
}

\author{
Juliana Tamie Yamada*; Sarah Nayumi Nishihira"; Márcia Torturela***
}

\section{Resumo}

Descrever o perfil de idosos internados em uma unidade de internação geriátrica de acordo com a sua funcionalidade e sua dependência no autocuidado e avaliar sua evolução quanto a esses aspectos durante o período de internação. Os dados sociodemográficos e clínicos foram coletados por meio do histórico de enfermagem. $\mathrm{O}$ instrumento utilizado para realizar a avaliação da funcionalidade foi a Medida de Independência Funcional (MIF). A amostra foi composta por 31 idosos de idade superior ou igual a sessenta anos, que foram avaliados semanalmente quanto à sua funcionalidade para o autocuidado, desde a sua admissão até o momento de sua alta. $\mathrm{Na}$ avaliação da MIF autocuidado total houve uma redução da pontuação entre a primeira $(16,6)$ e terceira $(14,3)$ semana, porém sem significância estatística $(p=0,239)$. Analisando os itens da MIF separadamente (alimentação, higiene, banho, uso do vaso sanitário e vestir-se aci- ma e abaixo da cintura), houve redução da pontuação com significância estatística na maioria dos itens durante o período de internação. Houve uma tendência à piora na funcionalidade do idoso com relação ao autocuidado durante o período de internação hospitalar. Portanto, a assistência de enfermagem deve criar condições que possibilitem ao idoso manter e implementar o autocuidado, para que adquira independência e conviva da melhor maneira possível com suas limitações, facilitando, dessa forma, sua reintrodução na sociedade e na família.

Palavras-chave: Saúde do idoso. Autocuidado. Funcionalidade.

* Aprimoranda de Enfermagem em Geriatria e Gerontologia do Hospital do Servidor Público. Endereço para correspondência: Rua Jardim de Repouso, 881, CEP 07261-000, Guarulhos - SP. E-mail: juzinhayamada@ hotmail.com.

** Fonoaudióloga especializada em Gerontologia pela Escola Paulista de Medicina; supervisora da pós-graduação em Gerontologia, da disciplina de Geriatria e Gerontologia da Universidade Federal de São Paulo.

*** Enfermeira supervisora de Enfermagem em Geriatria e Gerontologia do Hospital do Servidor Público Estadual; coordenadora e supervisora do curso de Extensão em Gerontologia do Hospital do Servidor Público Estadual. Local da Pesquisa: Hospital do Servidor Público Estadual. E-mail: juzinhayamada@hotmail.com

$\hookrightarrow$ Recebido em maio de 2009 - Aceito em maio de 2009.

$\longrightarrow$ doi:10.5335/rbceh.2009.024 


\section{Introdução}

O processo do envelhecimento e sua consequência natural, a velhice, é uma das preocupações da humanidade desde o início da civilização. Estima-se que no ano de 2025 o Brasil venha a se tornar a sexta maior população de idosos no mundo, com um percentual equivalente a $15 \%$ de indivíduos maiores de sessenta anos em relação aos demais. (IBGE, 2000). Ainda é grande a desinformação sobre a saúde do idoso e as particularidades e desafios do envelhecimento populacional.

Segundo Freitas et al. (2006), poucos problemas têm merecido tanta atenção e preocupação do homem como o envelhecimento e a incapacidade funcional associada a esse processo. Ramos e Neto (2005) referem que, diante do envelhecimento da população idosa, há a necessidade de estruturação de serviços e programas de saúde que possam responder às demandas emergentes do novo perfil epidemiológico do país. De forma geral, percebe-se um aumento no número de internações hospitalares entre os idosos, relacionado tanto com a senescência quanto com a senilidade.

A avaliação de idosos centrada na enfermagem, segundo Lueckenotte (2002), deve ser fundamentada nos conhecimentos gerontológicos. Independentemente da estrutura ou do instrumento utilizado, o profissional de enfermagem deve coletar os dados enquanto observa os seguintes princípios: utilização de uma abordagem individual centrada na pessoa, consideração ao cliente como participante no controle e no tratamen- to de sua saúde e ênfase na capacidade funcional.

Visto que a maior parte desta população é acometida por doenças crônicodegenerativas, somada à decorrência de pluripatogenia (evidência de mais de uma doença concomitante), podem ser consideradas responsáveis pela necessidade de maior permanência hospitalar e pela progressiva perda de autonomia dos idosos. (SALES; SANTOS, 2007).

As doenças diagnosticadas num indivíduo idoso geralmente não admitem cura e, se não forem devidamente tratadas e acompanhadas ao longo dos anos, tendem a apresentar complicações e sequelas que comprometem a independência e a autonomia do paciente. A saúde não é mais medida pela presença ou não de doenças, e sim pelo grau de capacidade funcional. (RAMOS, 2003).

Para Orem (1980), o autocuidado é a prática de atividades que o indivíduo inicia e executa em seu próprio benefício na manutenção da vida, da saúde e do bem-estar. Tem como propósito as ações que, seguindo um modelo, contribuem de maneira específica para a integridade das funções e o desenvolvimento humano.

Quando um paciente apresenta déficit no autocuidado, a enfermagem mostra o quanto é necessário. Após o enfermeiro identificar esse déficit, estabelece o plano de ação junto ao paciente, delegando a sua responsabilidade, a do paciente e de outros profissionais para que a demanda terapêutica do autocuidado seja atendida. (OREM, 1980).

O envelhecimento populacional é uma realidade e requer atendimento especializado. Como a incidência de in- 
ternações hospitalares em enfermarias geriátricas é alta, resolvemos averiguar a funcionalidade de acordo com a sua dependência no autocuidado durante a internação. Julgamos que desse modo seria possível a definição de estratégias e um caminho que vise à melhoria contínua da qualidade da assistência prestada.

\section{Metodologia}

Trata-se de um estudo quantitativo e descritivo realizado na enfermaria geriátrica do Hospital do Servidor Público Estadual Francisco Morato de Oliveira, com 31 indivíduos de idade superior ou igual a sessenta anos, que foram avaliados semanalmente quanto à sua funcionalidade para o autocuidado, desde a sua admissão até o momento de sua alta. Todos os indivíduos ou seus responsáveis assinaram o termo de consentimento livre e esclarecido. Foram excluídos da amostra os pacientes com menos de sete dias de internação.

Os dados sociodemográficos e clínicos foram coletados por meio do histórico de enfermagem, onde constam dados de identificação, hipóteses diagnósticas, além de dados específicos da enfermagem, o qual foi aplicado no ato da internação pela avaliadora. $\mathrm{O}$ instrumento utilizado para realizar a avaliação da funcionalidade foi a Medida de Independência Funcional (MIF), validado no Brasil por Riberto et al. (2004). Sua aplicação foi feita com o paciente e/ou seu cuidador/responsável; quando isso não era possível, foi preenchido de acordo com a observação realizada pela pesquisadora.
Trata-se de um instrumento de avaliação da incapacidade de pacientes com restrições funcionais de origem variada, tendo sido desenvolvido na América do Norte na década de 1980. Seu objetivo primordial é avaliar de forma quantitativa a carga de cuidados demandada por uma pessoa para a realização de uma série de tarefas motoras e cognitivas de vida diária. Entre as atividades avaliadas estão o autocuidado, controle esfincteriano, transferências, locomoção, comunicação e cognição social. Cada uma dessas atividades recebe uma pontuação de de 1 (dependência total) a 7 (independência completa), com total que varia de 18 a 126 pontos.

Para este estudo foram utilizadas apenas as atividades para $o$ autocuidado alimentação (AL), higiene (H), banho (B), vestir-se acima da cintura (PC), vestirse abaixo da cintura (PD) e uso do vaso sanitário (BANH), obtendo-se uma pontuação que variou de 6 a 42 pontos.

Este trabalho foi aprovado pelo Comitê de Ética em pesquisa do Instituto de Assistência Médica do Servidor Público Estadual (IAMSPE) sob o $\mathrm{n}$. $121 / 08$. Os dados obtidos foram tabulados e analisados estatisticamente pelo programa SPSS versão 14.0. Foi considerado estatisticamente significante valor de $\mathrm{p}$ menor que 0,05 e utilizada análise de variância (Anova) com medidas repetidas.

\section{Análise e discussão dos dados}

Foram avaliados 31 idosos com idade superior ou igual a sessenta anos. Verificou-se que a maioria dos pacientes 
internados tinha idade acima de oitenta anos $(66,1 \%)$, caracterizando uma população da quarta idade. Segundo Moraes e Costa (2007), a expectativa de vida vem aumentando e cada vez mais encontramos pessoas com idade superior a oitenta anos. A maioria da população analisada é do sexo feminino (90\%). Todos os pacientes tinham ao menos uma doença crônica, como diabete melitus, doença pulmonar obstrutiva crônica, acidente vascular cerebral, demências, cardiopatias, hipertensão arterial sistêmica, broncopneumonia e insuficiência renal crônica. Dentre as comorbidades encontradas as principais foram hipertensão $(90,3 \%)$ e cardiopatias $(54,8 \%)$, como mostra a Figura 1. A média do tempo de internação do estudo foi de 14 dias, com ampla variação (7 a 56 dias).

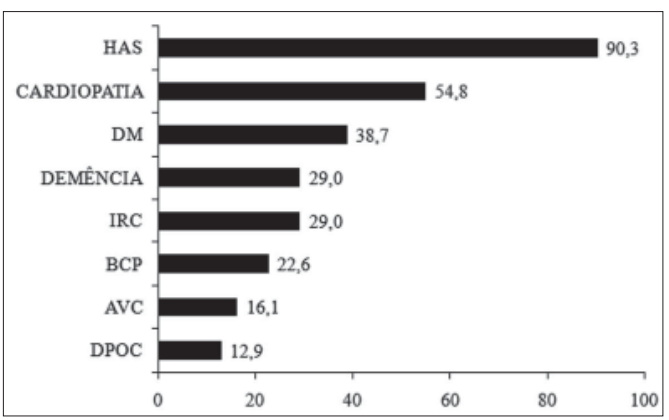

Figura 1 - Comorbidades encontradas.

A faixa etária mais frequente encontrada neste trabalho, entre 80-90 anos, como mostra a Figura 2, condiz com dados da literatura, já que muitos estudiosos da área relatam o crescimento da expectativa de vida. Conforme Bee (1997), a tendência na expectativa de vida dos seres humanos durante o último milênio tem sido caracterizada por um aumento lento, mas gradual, interrompido por uma votabilidade no índice de mortalidade por epidemias e pandemias de doenças infecciosas, fome e guerras. Essa votabilidade foi drasticamente reduzida na metade do século XIX, à medida que essas doenças infecciosas foram sendo combatidas eficazmente e houve melhoria das condições de vida, como também de políticas de saúde. Cientistas afirmam que o que comanda o envelhecimento é o ambiente e o estilo de vida, sendo a genética de menor influência dentre esses. Ainda que existam genes que estão envolvidos no processo de envelhecimento, é o ambiente o fator determinante da longevidade.

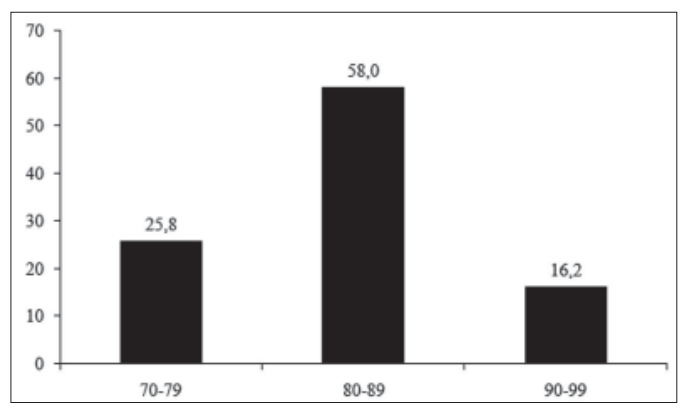

Figura 2 - Distribuição da faixa etária.

Com relação ao sexo Parahyba, Veras e Melzer (2005) explicam que as mulheres não desenvolvem incapacidades funcionais com maior frequência do que os homens, mas sobrevivem mais tempo do que eles com suas limitações. Motta (1999) explica essa diferenciação das maneiras de viver a velhice por critérios combinados de gênero e classe social: mulheres de classe baixa e média tendem a se afirmar pela atividade e pela participação em espaços de lazer e con- 
vivência, os quais usam como símbolos de liberdade, muitas vezes até ultrapassando os limites das convenções sociais; por sua vez, as mulheres de classe média tendem a ir para as universidades, que lhes oferecem a oportunidade de se atualizarem e de aprenderem sobre o mundo e sobre si próprias, num contexto a que sempre sonharam pertencer.

Em contrapartida, os homens de classe média e baixa tendem a se congregar mais em federações e confederações de pensionistas e aposentados para lutar por seus direitos. A feminização da velhice também é indicada pelo crescimento relativo da taxa de mulheres idosas que são chefes de família e que fazem parte da população economicamente ativa.

Zaslavsky e Gus (2002) relatam que, atualmente, o que preocupa no idoso é a sua saúde global, tornando as comorbidades importantes, e as estatísticas mostram que a maior causa de mortalidade e morbidade é a doença cardiovascular. A doença coronariana é a causa de 70 a $80 \%$ de mortes tanto em homens como em mulheres e a insuficiência cardíaca congestiva, mais comum de internação hospitalar, de morbidade e mortalidade na população idosa. Em virtude desses dados, fica evidente que cuidados com as comorbidades e um programa direcionado à prevenção primária no idoso permitirão que no futuro as doenças degenerativas e, fundamentalmente, as cardiovasculares possam ser bem atenuadas.

A Figura 3 mostra a distribuição de raça, mas não foram encontrados estudos que possam justificar tal predominância.

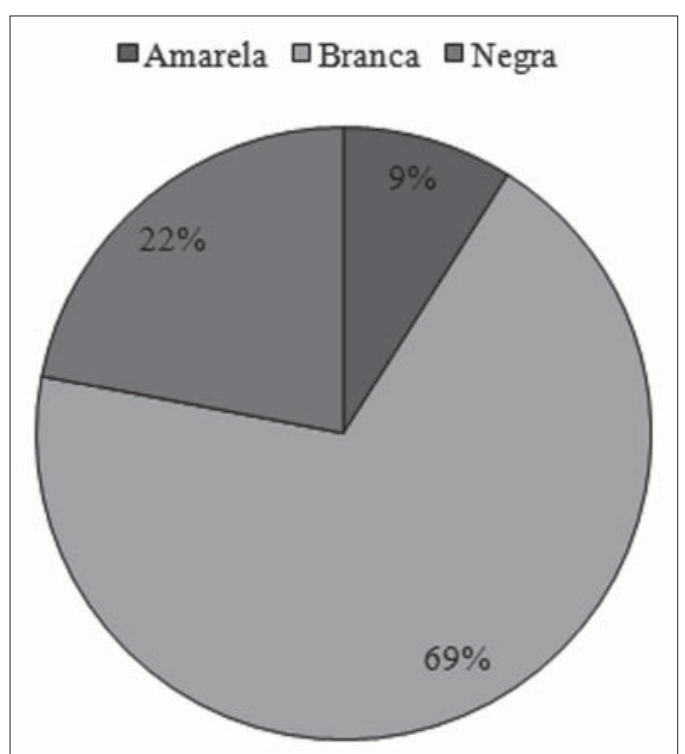

Figura 3 - Distribuição de raça.

Durante o processo de internação hospitalar foi observado que todos possuíam algum tipo de alteração visual e algum déficit auditivo, o que traz prejuízos também para uma boa capacidade funcional do idoso.

Foi observado que a funcionalidade dos indivíduos internados na enfermaria geriátrica já era, de certa forma, prejudicada em razão das suas comorbidades e que no decorrer da internação houve uma tendência a piorar, o que pode ser justificado pela própria internação, visto que aumentam os riscos à exposição ambiental, com o consequente risco de infecção hospitalar.

Para análise estatística da MIF foram estudados apenas pacientes até a terceira semana de internação (n = 14), em virtude da redução do número de pacientes após este período. Foi determinada a média dos valores da MIF 
total do autocuidado e de cada item que o compõe durante as três primeiras semanas e estudada sua variação. Na avaliação funcional, percebeu-se que na MIF total do autocuidado houve tendência à redução da pontuação, porém sem significância estatística $(p=0,239)$. Quando realizadas comparações múltiplas de mínimos quadrados, também não foram encontradas relações significantes. Para melhor visualizar as diferenças entre os momentos apresentam-se na Figura 4 os intervalos de confiança para a média dos valores para autocuidado.

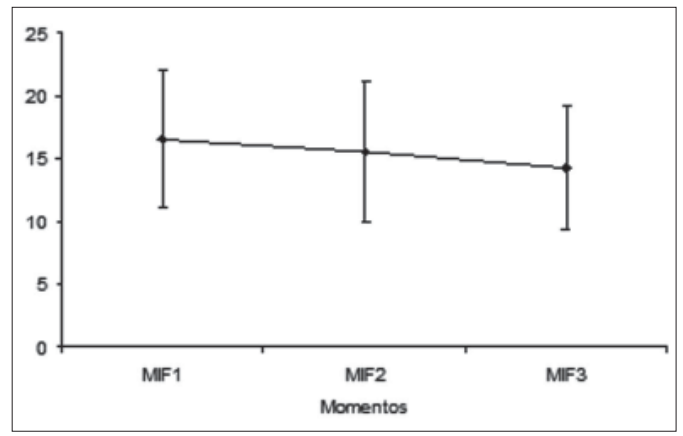

Figura 4 -Intervalo de confiança para a média dos valores para autocuidado.

Na análise da MIF no autocuidado avaliado separada e semanalmente no item alimentação, verifica-se que houve uma redução na pontuação (Tab. 1) com significância estatística entre os momentos. Quando aplicadas comparações múltiplas de mínimos quadrados, temse a Tabela 2, que fornece os valores de (p) para as comparações 2 a 2 entre os momentos.
Tabela 1 - Médias e desvios-padrão. MIF - AL (Alimentação).

\begin{tabular}{l|c|c|c}
\hline \multicolumn{1}{c|}{ Estatísticas } & AL1 & AL2 & AL3 \\
\hline Média & 4,07 & 3,57 & 3,36 \\
Desvio-padrão & 1,82 & 1,99 & 1,91 \\
$\mathrm{~N}$ & 14 & 14 & 14 \\
\hline
\end{tabular}

Anova. $\mathrm{p}<0,0001^{*}$

Tabela 2 - Valores de p.

\begin{tabular}{c|c|c}
\hline & Inicial & 2 \\
\hline 2 & 0,0537 & \\
3 & $<0,0001^{*}$ & $0,0003^{*}$ \\
\hline
\end{tabular}

Inicial $=1$ semana $<2$ semanas

Para melhor visualizar as diferenças acima apresentam-se na Figura 5 os intervalos de confiança para a média do item alimentação.

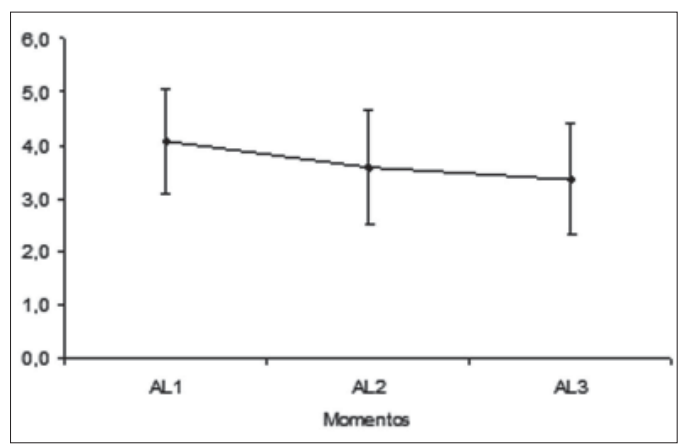

Figura 5 - Intervalo de confiança para a média do item alimentação.

No item higiene houve redução da pontuação (Tab. 3) com diferença significante entre os momentos. Nas comparações múltiplas de mínimos quadrados a Tabela 4 fornece os valores de (p) para as comparações 2 a 2 entre os momentos. 
Tabela 3 - Médias e desvios-padrão MIF - H (Higiene).

\begin{tabular}{l|c|c|c}
\hline \multicolumn{1}{c|}{ Estatísticas } & $\mathrm{H} 1$ & $\mathrm{H} 2$ & $\mathrm{H} 3$ \\
\hline Média & 3,57 & 3,21 & 3,07 \\
Desvio-padrão & 2,17 & 2,16 & 1,90 \\
$\mathrm{~N}$ & 14 & 14 & 14 \\
\hline
\end{tabular}

Anova. $\mathrm{p}<0,0001^{*}$.

Tabela 4 - Valores de p.

\begin{tabular}{c|c|c}
\hline & Inicial & 2 \\
\hline 2 & 0,0654 & \\
3 & $<0,0001^{*}$ & $0,003^{*}$ \\
\hline
\end{tabular}

Inicial $=1$ semana $<2$ semanas.

Para melhor visualizar as diferenças acima apresentamos na Figura 6 os intervalos de confiança para a média do item higiene.

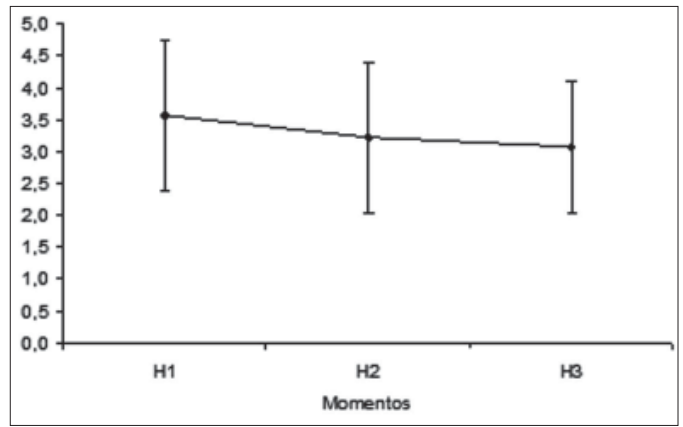

Figura 6 -Intervalo de confiança para a média do item higiene.

No item banho houve redução da pontuação, porém sem diferença significante entre os momentos ( $p=0,124)$. Mesmo não significante ao aplicar comparações múltiplas de mínimos quadrados, a Tabela 5 fornece os valores de (p) para as comparações 2 a 2 entre os momentos.
Tabela 5 - Valores de p.

\begin{tabular}{c|c|c}
\hline & Inicial & 2 \\
\hline 2 & 0,162 & \\
3 & $0,047^{\star}$ & 0,546 \\
\hline \multicolumn{2}{l}{ Anova. $\mathrm{p}=0,124}$.
\end{tabular}

Para melhor visualizar as diferenças acima apresentam-se na Figura 7 os intervalos de confiança para a média do item banho.

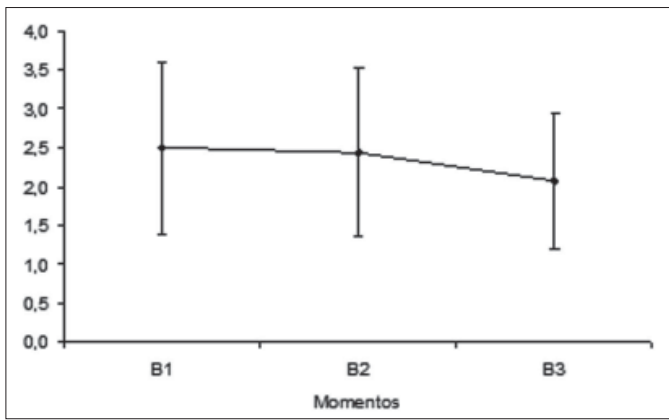

Figura 7 - Intervalo de confiança para a média do item banho.

No item vestir-se acima da cintura (PC) houve redução da pontuação com significância estatística (Tab. 6). Para comparações múltiplas de mínimos quadrados a Tabela 7 apresenta os valores de (p) para as comparações 2 a 2 entre os momentos.

Tabela 6 - Médias e desvios-padrão MIF - PC (Vestir-se acima da cintura).

\begin{tabular}{l|c|c|c}
\hline \multicolumn{1}{c|}{ Estatísticas } & PC1 & PC2 & PC3 \\
\hline Média & 2,29 & 2,14 & 1,93 \\
Desvio-padrão & 1,73 & 1,66 & 1,49 \\
$\mathrm{~N}$ & 14 & 14 & 14 \\
\hline
\end{tabular}

Anova. $p=0,0006^{*}$. 
Tabela 7 - Valores de p.

\begin{tabular}{c|c|c}
\hline & Inicial & 2 \\
\hline 2 & $0,0326^{*}$ & \\
3 & $0,0001^{*}$ & 0,059 \\
\hline
\end{tabular}

Inicial $>1$ semana $=2$ semanas.

Para melhor visualizar as diferenças acima apresentam-se na Figura 8 os intervalos de confiança para a média vestir-se acima da cintura.

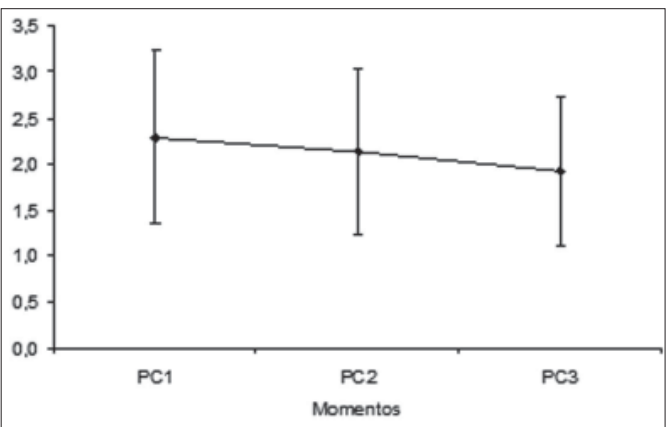

Figura 8 -Intervalo de confiança para a média vestir-se acima da cintura.

No item vestir-se abaixo da cintura (PD) houve redução da pontuação com diferença significante entre os momentos (Tab. 8). Aplicando comparações múltiplas de mínimos quadrados, temse a Tabela 9, que fornece os valores de (p) para as comparações 2 a 2 entre os momentos.

Tabela 8 - Médias e desvios-padrão MIF - PD (Vestir-se abaixo da cintura).

\begin{tabular}{l|c|c|c}
\hline \multicolumn{1}{c|}{ Estatísticas } & PD1 & PD2 & PD3 \\
\hline Média & 2,00 & 2,14 & 1,93 \\
Desvio-padrão & 1,57 & 1,66 & 1,49 \\
$\mathrm{~N}$ & 14 & 14 & 14 \\
\hline
\end{tabular}

Anova. $\mathrm{p}=0,003^{*}$.
Tabela 9 - Valores de p.

\begin{tabular}{c|c|c}
\hline & Inicial & 2 \\
\hline 2 & 0,263 & \\
3 & $0,0009^{*}$ & $0,021^{*}$ \\
\hline
\end{tabular}

Inicial $=1$ semana $>2$ semanas

Para melhor visualizar as diferenças acima apresentam-se na Figura 9 os intervalos de confiança para a média do item vestir-se abaixo da cintura.

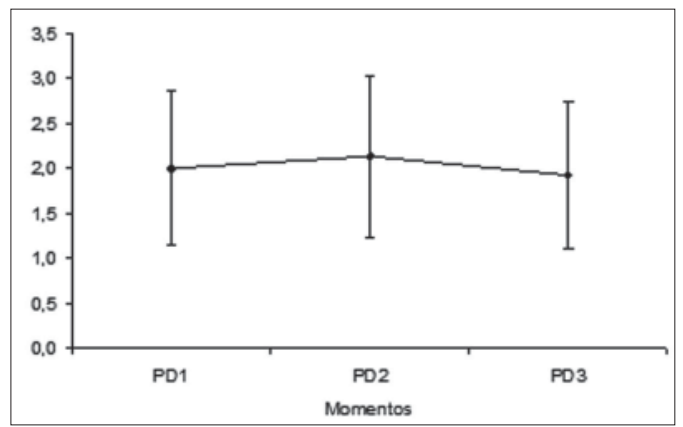

Figura 9 - Intervalo de confiança para a média do item vestir-se abaixo da cintura

No item uso do vaso sanitário houve redução da pontuação com diferença significante entre os momentos (Tab. 10). Ao analisar comparações múltiplas de mínimos quadrados, a Tabela 11 fornece os valores de (p) para as comparações 2 a 2 entre os momentos.

Tabela 10 - Médias e desvios-padrão MIF BANH (Uso do vaso sanitário).

\begin{tabular}{l|c|c|c}
\hline \multicolumn{1}{c|}{ Estatísticas } & BANH1 & BANH2 & \\
\hline Média & 2,07 & 2,14 & 1,57 \\
Desvio-padrão & 1,69 & 1,88 & 1,45 \\
$\mathrm{~N}$ & 14 & 14 & 14 \\
\hline
\end{tabular}

Anova. $\mathrm{p}=0,0007^{*}$.

Tabela 11 - Valores de p.

\begin{tabular}{c|c|c}
\hline & Inicial & 2 \\
\hline 2 & 0,229 & \\
3 & $0,0002^{\star}$ & $0,008^{\star}$ \\
\hline
\end{tabular}

Inicial $=1$ semana $>2$ semanas. 
Para melhor visualizar as diferenças acima apresentam-se na Figura 10 os intervalos de confiança para a média do item uso do vaso sanitário.

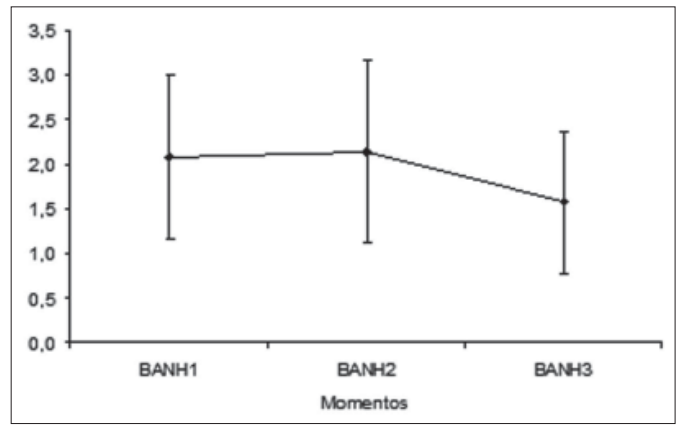

Figura 10 - Intervalo de confiança para a média do item uso do vaso sanitário

\section{Discussão}

Observou-se que os idosos internados apresentaram tendência a um declínio de sua capacidade funcional, o que traz dificuldades para intervenções junto a estes indivíduos, havendo necessidade de avaliação da assistência prestada a eles pelos serviços de saúde pública.

Ao observar e considerar todas as limitações dos idosos, a MIF pode ser considerada como um marcador de saúde de grande serventia, uma vez que as funções humanas básicas são determinantes para a habilidade do autocuidar. A avaliação de cada função mostra se o idoso tem capacidade para ser independente para o autocuidado ou se necessita de ajuda. Dessa forma, o trabalho demonstra que a prática geriátrica deve ser interdisciplinar, na qual cada profissional tem papel fundamental para a melhora do estado funcional do idoso.

De forma especial, uma maior estimulação ao idoso por parte da equipe de enfermagem deve ser realizada, a qual tem como princípio de seu trabalho o cuidar, a manutenção e melhoria da funcionalidade, prevenção e promoção da saúde, recuperação e reabilitação de idosos que venham a ter comprometida sua capacidade funcional. Ainda, segundo Santos (2000), o melhor local para se desenvolver o cuidado a um ser humano idoso é a sua casa, junto a sua família e na sua comunidade. A hospitalização e institucionalização do idoso devem ser evitadas o máximo possível.

\section{Conclusão}

Observou-se que houve uma tendência na piora da funcionalidade do idoso com relação a autocuidados numa enfermaria geriátrica durante o período de internação. A assistência de enfermagem deve criar condições que possibilitem ao idoso manter e implementar o autocuidado, levando em consideração a individualidade de cada um, para que adquiram independência e convivam da melhor maneira possível com suas limitações, facilitando dessa forma sua reintrodução na sociedade e na família.

Application of Functional Independence Measure in the practice of self care in a geriatric ward

\section{Abstract}

To describe the profile of older people admitted to a hospital geriatric unit in accordance with its function and its dependence on self care and assess their progress on these aspects during the hospitalization. The socio-demographic data and clinical trials were collected through the history of nursing. The instrument used to perform the assessment of functionality was the Functional Independence Measure (FIM). The 
sample was composed of 31 elderly people of age superior or equal than 60 years, who were assessed weekly, as to its functionality for self care, from their admission until the time of his discharge. In the assessment of self care MIF total scores there was a reduction between the $1 \mathrm{st}(16,6)$ and $3 \mathrm{rd}$ $(14,3)$ weeks, but without statistical significance $(p=0,239)$. Analyzing the items of MIF separately (food, hygiene, bathing, using the toilet and put it above and below the belt) there was a decrease in the scores of MIF with statistical significance in most of the items during the period of hospitalization. There was a tendency to decrease the functionality of the elderly with respect to self-care during the hospitalization. So the nursing care must create conditions that allow the elderly to maintain and implement the self care, so that they become independent and live the best possible way with their limitations, facilitating thus their reintroduction into society and family.

Key words: Health of the elderly. Self care. Functionality.

\section{Referências}

BEE, H. O ciclo vital. Porto Alegre: Artes Médicas, 1997.

FREITAS, E. et al. Tratado de geriatria e gerontologia. 2. ed. Rio de Janeiro: Guanabara Koogan, 2006.

IBGE. Anuário Estatístico do Brasil. Censo demográfico 2000. Disponível em: <www. ibge.gov.br/home/estatistica/populacao/censo2000>. Acesso em: 28 abr. 2009.

LUECKENOTTE, A. G. Avaliação em gerontologia. 3. ed. Rio de Janeiro: Reichmann \& Affonso Editores, 2002.

MOTTA, A. B. As dimensões de gênero e classe social na análise do envelhecimento. Cadernos Pagu, Campinas, v. 13, p. 191-221, 1999.
MORAES, D. S.; COSTA, M. R. N. Quarta idade: será possível envelhecer com dignidade? Disponível em: <ftp://74.125.113.132/search ?q=cache:Ec2XqdzaPvQJ:guaiba.ulbra.tche. br/pesquisas/2007/artigos/psicologia/265.pdf +quarta+idade+ser\%C3\%A1+poss $\% \mathrm{C} 3 \% \mathrm{ADv}$ el\&hl=pt-BR\&ct=clnk\&cd=1\&gl=br\&client= firefox-a>. Acesso em: 23 mar. 2009.

OREM, D. E. Nursing: concepts of practice. 2. ed. New York: McGrau-Hill, 1980.

PARAHYBA, M. I.; VERAS, R.; MELZER, D. Incapacidade funcional entre as mulheres idosas no Brasil. Revista de Saúde Pública, São Paulo, v. 43, n. 5, p. 796-805, 2005.

RAMOS, L. R.; NETO, J. T. Geriatria e gerontologia. São Paulo: Manole, 2005.

RAMOS, L. R. Fatores determinantes do envelhecimento saudável em idosos residentes em centro urbano. Projeto Epidoso, São Paulo. Cadernos Saúde Pública, Rio de Janeiro, v. 19, n. 3, p. 793-797, jun. 2003.

RIBERTO, M. et al. Validação da versão brasileira da medida de independência funcional. Acta Fisiátrica, v. 11, n. 2, p. 72-76, 2004.

SALES, F. M.; SANTOS, I. Perfil de idosos hospitalizados e nível de dependência de cuidados de enfermagem: identificação de necessidades. Texto Contexto - Enfermagem, Florianópolis, v. 16, n. 3, p. 495-502, 2007.

SANTOS, S. S. C. Enfermagem gerontológica: reflexão sobre o processo de trabalho. Revista Gaúcha de Enfermagem, Porto Alegre, v. 21, n. 2, p. 70-86, jul. 2000.

ZASLAVSKY, C.; GUS, I. Idoso, doença cardíaca e comorbidades. Arquivos Brasileiros de Cardiologia, Rio de Janeiro, v. 79, n. 6, p. 635-639, 2002. 